Original paper

\title{
Underestimation of dose delivery in preclinical irradiation due to scattering conditions
}

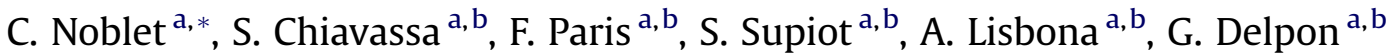 \\ a INSERM UMR 892, IRT UN, 8 quai Moncousu, 44007 Nantes, France \\ ${ }^{\mathrm{b}}$ ICO-Rene Gauducheau, Site Hospitalier Nord, Bd J. Monod, 44805 Saint Herblain, France
}

\section{A R T I C L E I N F O}

\section{Article history:}

Received 31 August 2012

Received in revised form

20 February 2013

Accepted 4 March 2013

Available online 26 March 2013

\section{Keywords:}

Dose monitoring

Scattering volume

AAPM-TG61

\begin{abstract}
A B S T R A C T
The aim of this study was to evaluate, by comparing simulation results with measurement results, the impact of the lack of scattering volume in experimental conditions of preclinical irradiation. First, a Monte Carlo model of a small animal irradiator, the Faxitron CP-160, was developed with GATE (Geant4 Application for Tomography Emission). To validate the model, simulated data were compared to depth dose and off-axis ratio profiles measured with a plane-parallel ionization chamber and Gafchromic ${ }^{\circledR}$ EBT films, respectively, in a solid water phantom. The AAPM TG-61 protocol was applied to measure the dose rate at the surface of a semi-infinite reference phantom. Then, the model was used to determine the dose distributions in three different phantom settings: a semi-infinite water phantom, a 2.8 -cm-thick water phantom and a $2.8-\mathrm{cm}$-diameter cylindrical water phantom. The dose distributions measured and simulated with Monte Carlo methods in a semi-infinite water phantom were similar $(<2 \%)$, thus validating our Monte Carlo model. The highest dose underestimation was observed between the reference and the cylindrical phantom (more than 15\% difference for the entrance dose) and was due to the lack of lateral scatter and backscatter. The use of standard backscatter factors and AAPM TG-61 protocol may result in a significant underestimation of the dose absorbed by small irradiated phantoms, such as mice or cells, in preclinical studies. Background: For preclinical radiotherapy studies, radiobiologists were used to determine the irradiation time depending only on the source surface distance. This work aimed to demonstrate that scatter conditions have a large impact on dose rate. Measurements and Monte Carlo simulations were used.
\end{abstract}

(c) 2013 Associazione Italiana di Fisica Medica. Published by Elsevier Ltd. All rights reserved.

\section{Introduction}

Preclinical radiotherapy studies aim at improving the efficiency of new protocols or new drugs by protecting healthy tissue or potentiating the antitumor effect of radiation. They are usually performed with dedicated irradiators on small animals such as mice and rats. Over the past 40 years, radioactive isotopes of cesium and cobalt have been the most commonly used sources of radiation for studies on cells or small animals. However, an increasing number of nuclear safety guidelines are being developed to limit their use. New directives also encourage the use of low or mediumenergy X-ray sources ( $100 \mathrm{kV}-400 \mathrm{kV})$. The penetrating power of these energy beams, unlike that of megavoltage beams used in clinical practice, is scaled to small animals. However, these beams

\footnotetext{
* Corresponding author. Tel.: +33 650713464 .

E-mail addresses: caroline.noblet@gmail.com (C. Noblet), Gregory.Delpon@ ico.unicancer.fr (G. Delpon).
}

are more absorbed by dense tissues such as bones because of the photoelectric effect being predominant in the kilovoltage range [1].

In experimental studies with small animal irradiators, the radiation dose is typically delivered by a static X-ray beam whose field diameter and dose rate only depend on source-surface distance. Moreover, due to poor placement accuracy, a single fraction is usually delivered, unlike clinical fractionated radiotherapy treatments. The whole body is irradiated, and in some cases, certain parts of the animal are shielded with a lead block. In clinical practice, thanks to the development of image-guided, intensitymodulated radiotherapy, the dose can be delivered in a more complex and accurate manner. When using small animal irradiators, clinical treatments are difficult to reproduce and the study of biological and physical inputs specific to new advances in radiation oncology is therefore limited.

Recently, several groups have tried to overcome this technical issue [2-8]. New small animal irradiators have been developed to mimic clinical conditions. The energy range is similar to that of previous preclinical irradiators and does not exceed $400 \mathrm{kV}$. 
But these devices deliver multiple precisely targeted beams of radiation with an image-guided system.

For low (below $100 \mathrm{kV}$ ) and medium-energy (100-300 kV) $\mathrm{X}$-rays, the AAPM TG-61 protocol is used to determine reference dose rate [9]. This protocol considers the absorbed dose at the surface of a full scatter water phantom based on in-air measurements and tabulated values of backscatter factors in water ( $\left.B_{\text {water }}\right)$. Nevertheless, in preclinical irradiations, the experimental scattering conditions are very different from these reference conditions. As a consequence, the tabulated backscatter values have to be corrected to take this difference into account [10,11].

The aim of this study was to evaluate the impact of the lack of lateral scatter and backscatter on the irradiation of mice with a medium-energy broad beam, with the purpose to improve the accuracy of absorbed dose determination for common preclinical irradiations. An X-ray irradiator used by the scientific community for radiobiological studies, i.e. the Faxitron CP-160 (Faxitron X-Ray Corp., Wheeling, IL, USA), was studied. A Monte Carlo model of this irradiator was created and validated by comparing data from simulations against measurements. Then, the model was used to evaluate the impact of scattering conditions on the dose distributions. Two different geometries were compared to an irradiation geometry under full scatter conditions $\left(30 \times 30 \times 30 \mathrm{~cm}^{3}\right.$ water phantom): (i) a $30 \times 30 \times 2.8 \mathrm{~cm}$ water phantom and (ii) a $2.8-\mathrm{cm}-$ diameter cylindrical water phantom. The impact of scattering conditions was also studied in solid phantoms to be able to compare simulations and measurements.

\section{Materials and methods}

\subsection{Faxitron $C P 160$}

The Faxitron CP-160 (Fig. 1) produces a $160 \mathrm{kVp}$ X-ray beam (6.3 $\mathrm{mA}$ ) adapted to the irradiation of small animals and cell cultures [12]. The system consists of a static X-ray tube with a $5 \mathrm{~mm}$ thick primary collimator having an aperture of $3.2 \mathrm{~cm}$ diameter. The X-ray tube (MXR-160, Comet, Switzerland) has a $0.8 \mathrm{~mm} \mathrm{Be}$ inherent filtration and a $0.5 \mathrm{~mm} \mathrm{Cu}$ additional filter.

In the shielded cabinet, an aluminum shelf can be positioned at different discrete levels. The distance from the source to the shelf varies from 12.7 to $99.0 \mathrm{~cm}$ (Table 1). The choice of source to surface distance (SSD) is a trade-off between the dose rate (and thus

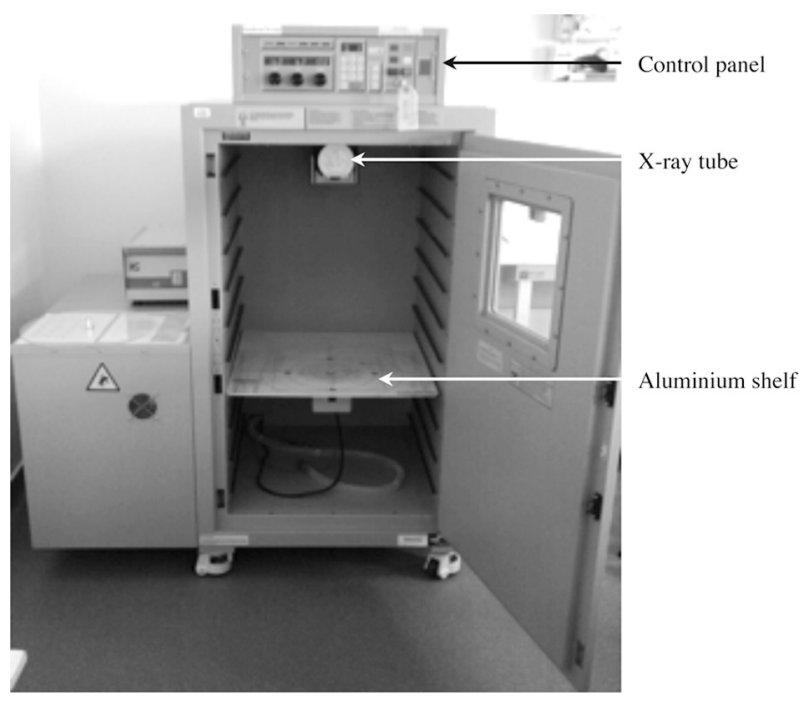

Figure 1. Faxitron CP 160 picture.
Table 1

Faxitron measured data $(160 \mathrm{kV}, 6.3 \mathrm{~mA}, 0.8 \mathrm{~mm}$ Be inherent filter and $0.5 \mathrm{~mm} \mathrm{Cu}$ additional filter) for the commonly used levels.

\begin{tabular}{llll}
\hline Level & SSD $(\mathrm{cm})$ & Field diameter $(\mathrm{cm})$ & Dose rate $(\mathrm{Gy} / \mathrm{min})$ \\
\hline 6 & 43.2 & 32.8 & 0.46 \\
7 & 33.0 & 25.1 & 0.78 \\
8 & 22.9 & 17.4 & 1.43 \\
9 & 12.7 & 9.6 & 3.91 \\
\hline
\end{tabular}

irradiation duration), the field diameter of the beam and the uniformity of the dose deposition in depth direction.

The Faxitron CP-160 only performs whole-body irradiation on small animals, although certain parts of the animal can be shielded with a lead block. The large beam leads to significant irradiation of the surrounding healthy tissues, unlike with clinical conformal techniques combining image-guided radiation therapy (IGRT) and intensity-modulated radiation therapy (IMRT).

\subsection{Commissioning of the Faxitron CP 160}

This section describes the dosimetric measurements performed to characterize the Faxitron CP-160 beam.

\subsubsection{Half-value layer}

Half-value layer (HVL) measurement was performed with a Farmer-type chamber (FC65-P, IBA, Germany) and a PTW UNIDOS electrometer (model 10002, PTW-Freiburg GmbH, Germany). A home-made cerrobend circular collimator was used to narrow the beam as recommended by TG61 protocol [9]. However, the source to ionization chamber distance was reduced to $30 \mathrm{~cm}$ due to the size of Faxitron unit. To determine the first HVL, $60 \mathrm{~s}$ irradiations were performed and dose was measured for different aluminum thicknesses varying from 0 to $11 \mathrm{~mm}$. The source to aluminum layers distance was approximately $15 \mathrm{~cm}$.

\subsubsection{Reference dosimetry}

The formalism in TG61 protocol enables the determination of absorbed dose at the surface of a phantom under full scatter conditions ( $\left.D_{\text {water, SSD }}\right)$. In this work, the in-air method was used for dose determination. This method is based on the Equation (1).

$$
D_{\text {water, } \operatorname{SSD}}=M_{\mathrm{air},} \mathrm{SSD}^{*} N_{\mathrm{k}} * B_{\mathrm{water}} \mathrm{SSD}^{*}\left[\left(\bar{\mu}_{\mathrm{en}} / \rho\right)_{\mathrm{air}}^{\text {water }}\right]_{\mathrm{air}}
$$

SSD is the source to phantom surface distance. $M_{\mathrm{air}, \mathrm{SSD}}$ is the free-in-air chamber reading (corrected for temperature, pressure, ion recombination and polarity effect). $N_{\mathrm{K}}$ is the air-kerma calibration coefficient obtained in air by PTW FREIBURG, for the beam closest to that of the Faxitron (kV/CDA: $150 \mathrm{kV} / 12.9 \mathrm{~mm} \mathrm{Al}$ ) in a $18 \times 18 \mathrm{~cm}^{2}$ field size. $B_{\text {water }}$ is the ratio of water kerma at the surface of a full scatter water phantom to water kerma at that point in the absence of the phantom. It depends on SSD, field diameter and HVL. $\left[\left(\bar{\mu}_{\mathrm{en}} / \rho\right)_{\mathrm{air}}^{\mathrm{water}}\right]_{\text {air }}$ is the ratio for water-to-air of the mean mass energy-absorption coefficients.

In this study, the in-air measurements were performed with a plane-parallel ionization chamber (type 233612, $30 \mathrm{~cm}^{3}$, PTWFreiburg GmbH, Germany) and a PTW UNIDOS electrometer (model 10002, PTW-Freiburg GmbH, Germany). Absorbed dose at the surface of a phantom under full scatter conditions was determined at each level from 6 to 9 (Table 1 ).

\subsubsection{Depth dose profiles}

Depth dose profiles were measured at $12.7,22.9$ and $33.0 \mathrm{~cm}$ SSDs corresponding respectively to $9.6,17.4$ and $25.1 \mathrm{~cm}$ field diameters. A plane-parallel ionization chamber (Roos chamber, 
model N34001, 0.35 cc, PTW-Freiburg GmbH, Germany) was used to limit the variation in the relative energy response, as recommended by Li et al. [13]. This chamber was inserted into an adapted RW3 slab (RW3, PTW-Freiburg GmbH, Germany). 120 s irradiations were performed and dose was measured at different depths ranging from 0 to $15 \mathrm{~cm}$.

\subsubsection{Off-axis dose profiles}

Off-axis dose profiles were measured at the surface of a 5-cmthick RW3 phantom. Gafchromic ${ }^{\circledR}$ EBT films (International Specialty Products, Wayne, NJ, USA) were used because the measurement of off-axis dose profiles with an ionization chamber within the Faxitron CP-160 was not convenient. The source to film distance was $17.9 \mathrm{~cm}$, corresponding to a field diameter of $13.6 \mathrm{~cm}$. Two orthogonal off-axis dose profiles were measured, in the anode-cathode direction and orthogonal to the anode-cathode direction. Twenty-four hours after irradiation, the film was digitized with an Epson V700 scanner (Epson America Inc., Long Beach, CA, USA) with the following parameters: 48bit color image type and a 72 dpi resolution. A calibration curve was previously produced to transform optical density into absolute dose by extracting the red channel of the film using ImageJ software (rsb. info.nih.gov/ij).

\subsection{Simulation of the Faxitron CP 160}

\subsubsection{Simulation code}

A Monte Carlo model of the Faxitron CP-160 has been developed with GATE v6.0 (Geant4 Application for Tomography Emission; www.opengatecollaboration.org), based on Geant4 libraries (v9.3). GATE was originally developed for positron emission tomography and single-photon emission computed tomography [14]. A recent release (v6.0) [15] allows performing simulations of external beam radiation therapy via the use of dose actors. A dose actor stores the dose deposited in a given volume into a 3D image according to the spatial position of the hits and takes into account the weight of the particles.

\subsubsection{Photon source}

A circular source of photons was simulated instead of accelerated electrons striking a tungsten target within the X-ray tube. SpekCalc software (Version 1.1) was used to calculate the photon spectrum [16]. Consequently, the heel effect was neglected in the model. This approximation was essential to reduce the simulation time.

\subsubsection{Particle tracking}

GATE provides different models for particle tracking. Each model includes specific processes and energy cuts. The standardenergy model, including electron ionization and multiple scattering above $1 \mathrm{keV}$, was selected for electron tracking. Below $1 \mathrm{keV}$, electrons were not tracked anymore and local energy deposit was thus considered. Indeed, the electron path does not exceed few microns in air at $1 \mathrm{keV}$, whereas in our simulations, the voxel dimensions were never less than $1 \times 1 \times 1 \mathrm{~mm}^{3}$. For photon tracking, the low-energy model was chosen: it takes into account the photon path down to $250 \mathrm{eV}$ and the Rayleigh process that occurs at very low energy levels.

\subsubsection{Half-value layer}

Simulations corresponding to the HVL measurements were performed with the Monte Carlo model for the different aluminum thicknesses. Dose actor was associated to an air box placed at $30 \mathrm{~cm}$ from the source. Three billion initial particles per simulation were generated.

\subsubsection{Depth dose profiles}

RW3 is currently used in clinical practice as water-equivalent material in the megavoltage energy range, although it has been shown not to be equivalent to water in the low and medium-energy ranges (up to $300 \mathrm{kVp}$ ) [17]. Consequently, RW3 material was simulated instead of water in the validation procedure of the Faxitron system. A RW3 phantom of $30 \times 30 \times 19 \mathrm{~cm}^{3}$ was modeled to represent the phantom used for ionization chamber measurements. The dose actor was associated to a $1 \times 1 \times 15 \mathrm{~cm}^{3}$ box whose surface was aligned with the RW3 phantom surface. The voxel dimensions for the output files were $1 \times 1 \times 0.2 \mathrm{~cm}^{3}$. To compare to ionization chamber measurements, three simulations were performed at SSDs of 12.7, 22.9 and $33.0 \mathrm{~cm}$, corresponding respectively to $9.6,17.4$ and $25.1 \mathrm{~cm}$ field diameters. One billion initial particles were generated for each simulation.

\subsubsection{Off-axis dose profiles}

A RW3 phantom of $30 \times 30 \times 5 \mathrm{~cm}^{3}$ was modeled. The dose actor was attached to a $30 \times 1 \times 0.1 \mathrm{~cm}^{3}$ box for the profile orthogonal to the anode-cathode direction and a $1 \times 30 \times 0.1 \mathrm{~cm}^{3}$ box for the profile in the anode-cathode direction. The surface of the box was aligned with the RW3 phantom surface. The source to surface phantom was $17.9 \mathrm{~cm}$, corresponding to a field diameter of $13.6 \mathrm{~cm} 1.5$ billion initial particles were generated.

\subsection{Impact of scattering conditions}

\subsubsection{Simulations in water}

Equation (1) does not take into account the lack of scatter when a small object such as a mouse is irradiated instead of a full scatter water phantom. To evaluate the impact of lack of lateral scatter and backscatter, two other irradiation geometries were simulated in addition to the full scatter phantom $\left(30 \times 30 \times 30 \mathrm{~cm}^{3}\right)$ : (i) a thinner water tank $\left(30 \times 30 \times 2.8 \mathrm{~cm}^{3}\right)$ to study only the lack of backscatter; and (ii) a $7 \mathrm{~cm}$-long water cylinder with a diameter of $2.8 \mathrm{~cm}$, representative of a mouse to evaluate the lack of both lateral scatter and backscatter. SSD was $19.1 \mathrm{~cm}$ and field diameter was $14.5 \mathrm{~cm}$. For each simulation, 1.5 billion initial particles were generated. Depth dose profiles in terms of $\mathrm{Gy} / \mathrm{mAs}$ were computed. (i) and (ii) surface dose rates and depth dose profiles were compared to the results obtained with the full scatter water phantom.

\subsubsection{Simulations and measurements in solid phantoms}

The impact of scattering conditions was also studied in solid phantoms to be able to compare simulations and measurements. A full scatter RW3 phantom $\left(30 \times 30 \times 30 \mathrm{~cm}^{3}\right)$ was simulated and two other irradiation geometries were studied: (i) a thin RW3 phantom $\left(30 \times 30 \times 2.8 \mathrm{~cm}^{3}\right)$; and (ii) a $7 \mathrm{~cm}$-long RMI 465 cylinder with a diameter of $2.8 \mathrm{~cm}$ (Gammex-RMI, Middleton, WI, USA). SSD was $19.1 \mathrm{~cm}$. For each simulation, 1.5 billion initial particles were generated. Simulated surface dose rates were compared to Gafchromic ${ }^{\circledR}$ EBT film measurements in the three different geometries. A calibration curve was produced to convert optical density into absolute dose.

\section{Results and discussion}

\subsection{Model validation}

\subsubsection{Half-value layer}

The measured and simulated first HVLs agreed within $0.5 \%$. They were $10.85 \mathrm{~mm} \mathrm{Al}$ and $10.92 \mathrm{~mm} \mathrm{Al}$, respectively. These results also agreed with the theoretical HVL calculated by SpekCalc software. In this study, we have considered a $0.8 \mathrm{~mm}$ Cu HVL corresponding to $11.0 \mathrm{~mm} \mathrm{Al}[9,16]$. 


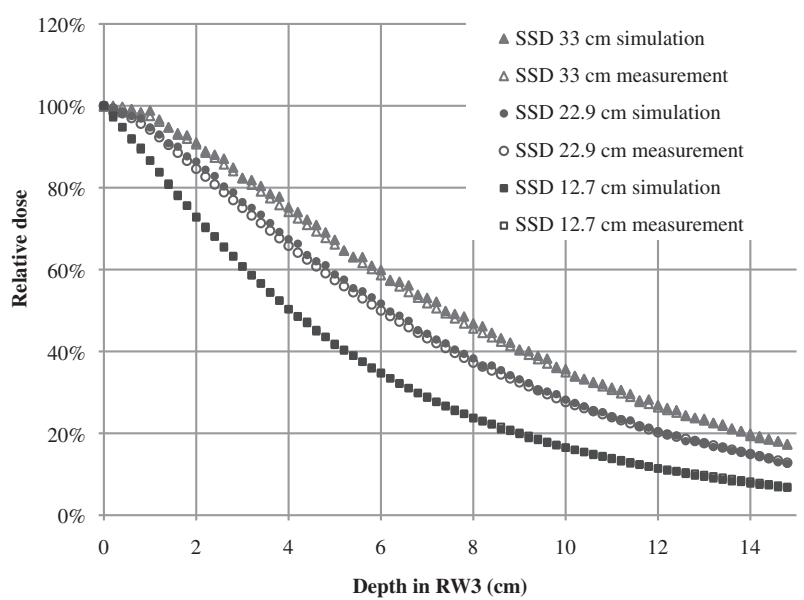

Figure 2. Percentage depth dose profiles for the $160 \mathrm{kVp}$ beam of the Faxitron CP-160 calculated using Monte Carlo methods and measured with a plane ionization chamber at different distances from the source. Statistical uncertainties associated with the presented results of the Monte Carlo calculations were below $1.8 \%$.

\subsubsection{Depth dose profiles}

The results of the comparison between simulated and measured depth dose profiles for the three different SSDs assessed are shown in Fig. 2. Profiles were normalized at the surface. The statistical uncertainty varied from $0.5 \%$ at the surface to $1.8 \%$ at a depth of $15 \mathrm{~cm}$. The simulated profiles showed a very good agreement with the measured depth dose profiles. Discrepancies between simulation and measurement were mainly less than $1.5 \%$, with a maximum difference of $2 \%$ observed at a depth of $4.2 \mathrm{~cm}$ for the SSD of $22.9 \mathrm{~cm}$. Attenuation decreased when SSD increased. The maximum dose was observed at the surface and then quickly decreased with depth in RW3. The 50 percent dose was observed at $7.4 \mathrm{~cm}, 6.0 \mathrm{~cm}$ and $4.0 \mathrm{~cm}$ in depth for the SSDs of $33.0 \mathrm{~cm}, 22.9 \mathrm{~cm}$ and $12.7 \mathrm{~cm}$, respectively.

The good agreement between simulated and measured profiles for the three SSDs indicated that the model was satisfactory.

\subsubsection{Off-axis dose profiles}

The results of the comparisons between simulated and measured off-axis dose profiles at the surface of a RW3 phantom are shown in Fig. 3. The homogenous area was defined as the area where the dose was higher than or equal to $90 \%$ of the central axis dose. The statistical uncertainty of the simulation was $0.6 \%$ in the homogenous

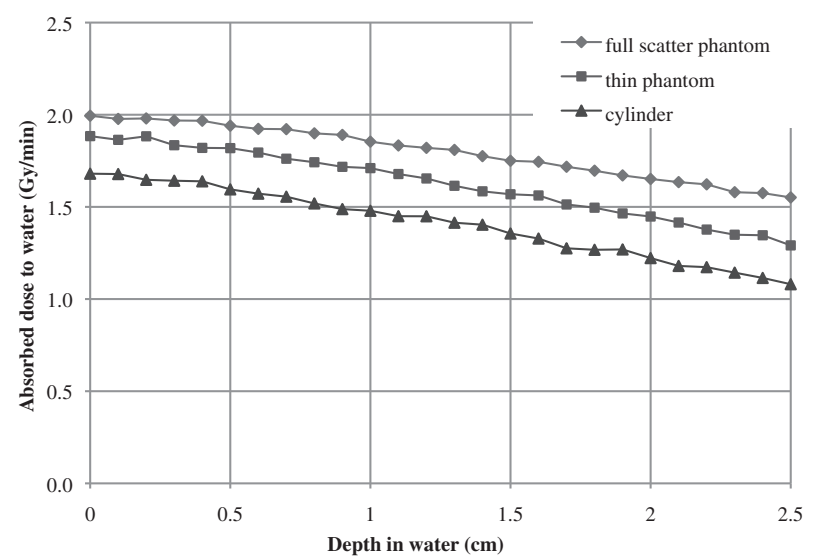

Figure 4. Depth dose profiles calculated using Monte Carlo methods with a full scatter water phantom, a non-full scatter water phantom (i) and a water cylinder (ii) at a SSD of $19.1 \mathrm{~cm}$ ( $14.5 \mathrm{~cm}$ field diameter).

area and reached up to $3 \%$ at $10 \mathrm{~cm}$ from the central beam axis. Along the anode-cathode axis (Fig. 3a), the homogenous area was approximately 8.9 and $9.5 \mathrm{~cm}$, respectively for the measured and simulated data. A large difference (up to $12 \%$ ) was observed between simulated and measured values along this axis, at approximately $4 \mathrm{~cm}$ from the central beam axis. This large difference is due to the heel effect, which results from the absorption of Bremsstrahlung photons by the tungsten target in the X-ray tube. The effect is neglected in the Monte Carlo simulations. Considering the small size of the irradiated objects (mice or cells), it could be assumed that the heel effect had a negligible impact on dose distribution in the homogenous area. Along the axis orthogonal to the anode-cathode direction (Fig. 3b), the homogenous area was approximately 9.2 and $9.5 \mathrm{~cm}$, respectively for the measured and simulated data. The heel effect had no impact on the profiles along this axis, where the relative difference between measured and simulated values was much lower. It never exceeded $2 \%(0.8 \%$ on average) in the homogenous area. Regardless of the axis, simulated and measured homogenous areas were similar. The model was satisfactory while irradiated objects are smaller than the homogenous area.

\subsection{Impact of scattering conditions}

Absorbed dose at the surface of a full scatter water phantom at SSD $19.1 \mathrm{~cm}$ was derived from absorbed dose calculated at SSD

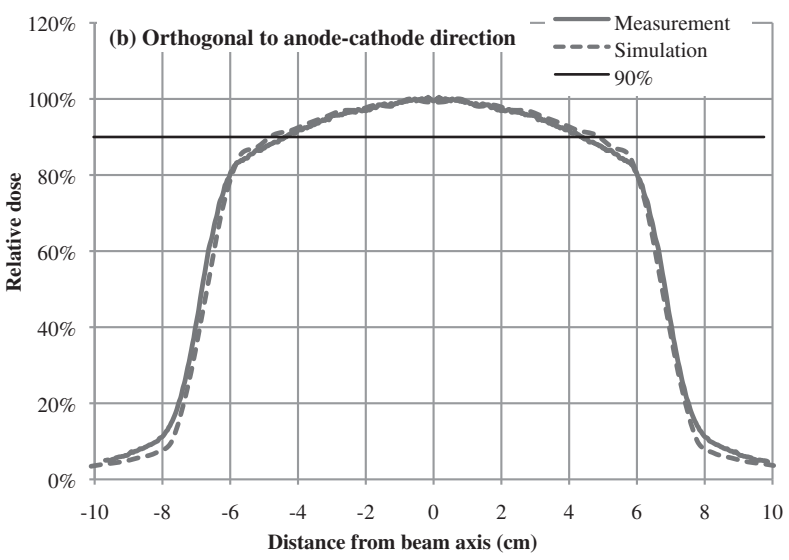

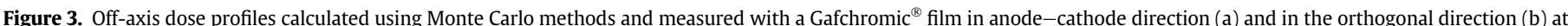

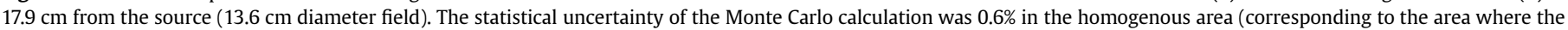
dose is higher than or equal to $90 \%$ of the central axis dose). 
Table 2

Simulated and measured dose rates at phantom surfaces (19.1 cm SSD and $14.5 \mathrm{~cm}$ field diameter).

\begin{tabular}{|c|c|c|c|c|}
\hline & & Full scatter phantom surface & Thin phantom surface & Cylinder surface \\
\hline \multirow[t]{3}{*}{ Simulation } & Dose rate $(\mathrm{Gy} / \mathrm{min})$ & 1.85 & 1.76 & 1.63 \\
\hline & Statistical uncertainty & $0.5 \%$ & $0.5 \%$ & $1.2 \%$ \\
\hline & Relative difference with full scatter phantom surface dose & - & $-4.8 \%$ & $-12.0 \%$ \\
\hline \multirow[t]{3}{*}{ Measurement } & Dose rate $(\mathrm{Gy} / \mathrm{min})$ & 1.82 & 1.82 & 1.63 \\
\hline & Standard deviation & $1.2 \%$ & $1.0 \%$ & $0.9 \%$ \\
\hline & Relative difference with full scatter phantom surface dose & - & $0.0 \%$ & $-10.4 \%$ \\
\hline Measurement/simulation difference & Relative difference & $1.6 \%$ & $-3.3 \%$ & $0.0 \%$ \\
\hline
\end{tabular}

$22.9 \mathrm{~cm}$ (level 8) according to Equation (1). A dose rate of $2.00 \mathrm{~Gy} / \mathrm{min}$ was obtained at SSD $19.1 \mathrm{~cm}$ by applying the inverse square law and taking into account the SSD and field diameter dependence of $B_{\text {water }}$. The corresponding simulation (full scatter phantom at SSD $19.1 \mathrm{~cm}$ ) led to the calculation of a conversion factor between Gy per incident particle to Gy per mAs. This factor was then applied to calculate Monte Carlo absolute dose rates.

\subsubsection{Simulations in water}

Figure 4 shows the simulated absolute depth dose profiles obtained with a full scatter water phantom, a $30 \times 30 \times 2.8 \mathrm{~cm}^{3}$ water phantom and a water cylinder.

The dose rate decreased faster with the thin phantom than with the full scatter phantom, because of the lack of backscatter in the former. The depth dose profiles obtained with the thin phantom and the cylinder were approximately parallel. The difference between absorbed dose in the full scatter phantom and the thin phantom was approximately $3.5 \%$ at the surface, and reached up to $14.5 \%$ in depth. The difference between absorbed dose in the full scatter phantom and the cylindrical phantom was $15 \%$ at the surface, and reached up to $30 \%$ in depth.

Consequently, the absorbed dose in water as determined by TG61 for the irradiation geometries not under full scatter conditions will be overestimated and the actual delivered dose will be less than the prescribed for these geometries.

\subsubsection{Simulations and measurements in phantoms}

Table 2 summarizes the results obtained for the simulated and the measured dose rates at the surfaces of the three different geometries. In the full scatter RW3 phantom, surface dose rate was 1.82 and $1.85 \mathrm{~Gy} / \mathrm{min}$ respectively for simulation and measurement. This result showed that RW3 is not water-equivalent since, at the same SSD and field diameter, the dose rate at the surface of a water phantom was $2.00 \mathrm{~Gy} / \mathrm{min}$. However there was a good agreement between simulated and measured results. The maximum difference was obtained for the thin phantom: dose rates differed by $3.3 \%$. These measurements confirmed the results observed by simulations in water. The impact of scattering conditions is not negligible for the calculation of the surface dose rate. If they are not taken into account, for small irradiated objects such as a mouse, dose underestimation may reach up to $10 \%$ depending on size.

\section{Conclusions}

The Faxitron Monte Carlo model was validated by comparing measured and simulated HVL and dose profiles in a full scatter phantom. Agreement was very satisfactory. Discrepancies were observed outside the homogenous area, especially along the anode-cathode axis because of the heel effect. It was assumed that the heel effect has no impact in experimental studies because irradiated objects are always positioned in the homogenous area.

Studies have been already published about $B_{\text {water }}$ factor variation for various tissue types [10] and for a particular experimental configuration [11] but a study about the impact of scattering volume on absorbed dose was still lacking. The Faxitron Monte Carlo model was used to evaluate the impact of scattering conditions in the determination of the surface dose rate. It has been shown with this work that surface dose and the variation of dose with depth depends of the overall size of the scattering volume along the beam axis but also laterally. If treatment times for a prescribed dose are calculated based on surface dose rates which are determined under full scatter conditions then the delivered dose to the irradiated specimen will be underestimated. The magnitude of error in the delivery of the prescription will depend on the dimensions of the animal being irradiated, perhaps also on the characteristics of the irradiation beam, and this, according to our results, could be of the order of at least $10 \%$. These results indicate that in preclinical studies on mice, the use of tabulated backscatter values may have an impact on biological conclusions, because the dose received may be significantly lower than the dose planned. Thus, the dose rate calculated with the AAPM TG61 protocol should be corrected to take into account the small animal volume. With Monte Carlo simulations, appropriate dose rates can be determined under experimental conditions. For preclinical studies, it would probably be very convenient to tabulate backscatter correction factors according to beam quality, SSD, field diameter and irradiated object size to take into account the scattering volume in the determination of the surface dose rate.

\section{Acknowledgments}

The authors would like to thank Nantes Metropole and the association "Ligue contre le cancer" for their financial support.

\section{References}

[1] Podgorsak E. Radiation oncology physics: a handbook for teachers and students international atomic energy agency, Vienna: 2009.

[2] Verhaegen F, Granton P, Tryggestad E. Small animal radiotherapy research platforms. Phys Med Biol 2011;56:R55-83.

[3] Clarkson R, Lindsay PE, Ansell S, Wilson G, Jelveh S, Hill RP, et al. Characterization of image quality and image-guidance performance of a preclinical microirradiator. Med Phys 2011;38(2):845-56.

[4] Wong J, Armour E, Kazanzides P, Iordachita I, Tryggestad E, Deng H, et al. A high resolution small animal research platform (SARRP) with X-ray tomographic guidance capabilities. Radiat Oncol Biol Phys 2008;71(5):1591-9.

[5] Strahinja S, Low DA. Hope AJ, Vicic M, Deasy JO, Cui J, et al. MicroRT - small animal conformal irradiator. Med Phys 2007;34(12):4706-43.

[6] Rodriguez M, Zhou H, Keall P, Graves E. Commissioning of a novel microCT/RT system for small animal conformal radiotherapy. Phys Med Biol 2009;54: 3727-40.

[7] Ngwa W, Korideck H, Chin LM, Makrigiorgos GM, Berbeco RI. MOSFET assessment of radiation dose delivered to mice using the Small Animal Radiation Research Platform (SARRP). Radiat Res 2011 Dec;176(6):816-20.

[8] Arndt CD, Wang IZ, Saito NG, Podgorsak MB. Dosimetric calibration and characterization for experimental mouse thoracic irradiation using orthovoltage X rays. Radiat Res 2011 Jun;175(6):784-9.

[9] Ma CM, Chair, Coffey CW, DeWerd LA, Liu C, Nath R, et al. AAPM protocol for $40-300 \mathrm{kV}$ X-ray beam dosimetry in radiotherapy and radiobiology. Med Phys 2001;28(6):868-93.

[10] Ma C-M, Seuntjens JP. Mass-energy absorption coefficient and backscatter factor ratios for kilovoltage X-ray beams. Phys Med Biol 1999;44:131-43.

[11] McKerracher C, Thwaites DI. Calibration of an X-ray cabinet unit for radiobiology use. Phys Med Biol 2006;51:3315-29. 
[12] Woo MK, Nordal RA. Commissioning and evaluating of a new commercial small rodent X-ray irradiator. Biomed Imaging Interv 2006;2(1).

[13] Li XA, Ma C-M, Salhani D. Measurement of percentage depth dose and lateral beam profile for kilovoltage X-ray therapy beams. Med Phys Biol 1997;42: 2561-8.

[14] Jan S, Santin G, Strul D, Staelens S, Assié K, Autret D, et al. GATE: a simulation toolkit for PET and SPECT. Phys Med Biol 2004;49:4543-61.
[15] Jan S, Benoit D, Becheva E, Carlier T, Cassol F, Descourt P, et al. GATE V6: a major enhancement of the GATE simulation platform enabling modelling of CT and radiotherapy. Phys Med Biol 2011;56:881-901.

[16] Poludniowski G, Landry G, DeBlois F, Evans PM, Verhaegen F. SpekCalc: a program to calculate photon spectra from tungsten anode X-ray tubes. Phys Med Biol 2009;54:N433-8.

[17] Hill R, Kuncic Z, Baldock C. The water equivalence of solid phantoms for low energy photon beams. Med Phys 2010;37(8):4355-63. 